\title{
Reopening the Doors to Center-Based ABA Services: Clinical and Safety Protocols During COVID-19
}

\author{
Julie Kornack ${ }^{1}$ (1) - Ashley L. Williams ${ }^{2} \cdot$ Katherine A. Johnson ${ }^{2} \cdot$ Eileen M. Mendes $^{3}$ \\ Published online: 30 June 2020 \\ (C) Association for Behavior Analysis International 2020
}

\begin{abstract}
In the wake of the coronavirus (COVID-19) pandemic, U.S. organizations that provide applied behavior analysis (ABA) programs to individuals with autism spectrum disorder have implemented a variety of safety precautions to minimize the spread of the virus, often shifting center-based services to the home or telehealth. Considered essential workers, ABA providers are exempt from government directives to close, so they have both the freedom and the great responsibility to make their own decisions about how best to keep their clients safe while continuing to provide medically necessary services. In the coming weeks and months, ABA providers will be faced with the decision about whether to reopen centers. This article does not address that decision, except to acknowledge the urgency to reopen, both to help clients and to remain solvent. Political rhetoric and contradictory public information further complicate this daunting decision. Because ABA providers do not have legal guidance to shift the burden of such decisions to local and state regulators, the burden is theirs alone. The unprecedented nature of the COVID-19 pandemic means that no decision is clearly wrong or right, and every decision has consequences. Although ABA providers do not have their own state guidance, many states have issued guidelines for childcare providers whose operations have continued throughout the pandemic. This article analyzes that guidance, identifies common variables potentially relevant to ABA organizations, highlights clinical considerations and procedural compliance, and provides ABA organizations with the tools to make the best decision for their clients, in their community, and on their timeline.
\end{abstract}

Keywords ABA $\cdot$ Center-based services $\cdot$ COVID-19 $\cdot$ Essential services $\cdot$ Health and safety $\cdot$ Pandemic

In the midst of an unprecedented pandemic and a growing understanding of COVID-19, applied behavior analysis (ABA) providers grapple with balancing the risk of exposing their clients and employees to the virus against the risk of withdrawing services and disrupting continuity of care (Cox,

Author Note This manuscript has not been previously published and has not been or will not be submitted elsewhere during the review process.

Electronic supplementary material The online version of this article (https://doi.org/10.1007/s40617-020-00462-7) contains supplementary material, which is available to authorized users.

Julie Kornack

J.Kornack@ centerforautism.com

1 Center for Autism and Related Disorders, 21600 Oxnard St., 18th floor, Woodland Hills, CA 91367, USA

2 LEARN Behavioral, Baltimore, MD, USA

3 Massachusetts Coalition of ABA Providers (MassCAP), Boston, MA, USA
Plavnick, \& Brodhead, 2020). Although, at the time of this publication, states are at different stages of shelter-at-home orders and business closure, health care providers, including behavior analysts, are viewed as essential workers and, therefore, exempt from such orders. As political and scientific leaders recognize that physical distancing will continue for some time and that a so-called second wave of COVID-19 diagnoses is likely (Sun, 2020), ABA providers may reevaluate temporary center-based closures and decide whether, when, and how to reopen. As providers come to understand the long-term suitability of their clients for telehealth services and encounter limits in home-based services, including employee and client concerns, the decision to reopen their centers will inevitably become part of the analysis of how best to facilitate access to ABA during a pandemic.

In advance of resuming center-based services, ABA providers are faced with a multitude of difficult and often conflicting considerations, ranging from clinical concerns to business solvency. Indefinitely delaying or limiting services may impose lifelong consequences on their clients and may 
exacerbate systemic staffing challenges. The potential to avoid or minimize these consequences has added weight in the hands of behavior analysts whose profession calls on them to shape behavior, including hygiene and physical-distancing behaviors that contribute to the safety of all involved.

Throughout this pandemic, many childcare providers have remained open to care for the children of essential workers. Elected officials have referenced the essential nature of childcare providers routinely and without controversy, and the Centers for Disease Control (CDC) and individual states have issued guidance for childcare providers. These guidance documents enumerate protocols to minimize the spread of the virus in settings that mimic the center-based settings of ABA providers, and existing research is rich with examples of ABA's effectiveness in teaching adherence to such protocols (e.g., Taylor \& Alvero, 2012). As such, common variables of the $\mathrm{CDC}$ and state guidance documents and relevant $\mathrm{ABA}$ protocols may offer useful insights into the considerations for resuming center-based services. Similarly, clinicians are urged to make use of technologies in ABA that would help prepare clients and staff for reentry into centers and begin preteaching skills that are relevant to the center-based setting. Each of these elements should be incorporated into a clear health and safety plan that has been carefully considered in light of state and federal guidance, aspects of the physical space, and the needs of staff and clients in order to successfully operate an $\mathrm{ABA}$ clinic during the continued presence of COVID-19. A health and safety plan template for reopening is provided in Figure 1.

As researchers and medical professionals learn more about the COVID-19 virus, available information continues to evolve. ABA organizations are encouraged to exercise an abundance of caution and do their own due diligence in ensuring the health, safety, and well-being of their clients, families, and employees. Guidance referenced herein is current as of the writing of this article.

\section{Analyzing Federal and State Childcare Guidance}

The CDC issued federal guidance specific to childcare facilities that have remained open during the pandemic (CDC, 2020a). The CDC guidance contemplates the essential nature of the childcare providers in that other essential workers who rely on childcare must be able to access childcare throughout the emergency. As a result, the CDC guidance does not mandate; it recommends and encourages specific safety precautions and qualifies most recommendations with "if possible" (CDC, 2020a). Many states reference or echo the CDC guidance in their own childcare guidance. See Table 1 for a listing of states' guidance.
States have broad authority to act to protect public health and safety (U.S. Const. amend. X). State guidance may be stricter than the federal guidance as long as the state guidance does not conflict with the federal guidance. Where state and federal guidance differ, the stricter guidance governs. Consequently, some states may require elements of the $\mathrm{CDC}$ guidelines that are only recommended in the federal guidance. Some states did not issue their own guidance and directed childcare workers to the CDC guidance (e.g., Alaska; CDC, 2020a). Other states referenced the CDC guidance and added their own requirements or suggestions (e.g., Massachusetts). Similarly, local officials may have also provided community-level guidance that may be stricter than the state or federal guidance as long as it does not conflict with state or federal guidance.

Similar to ABA providers, childcare providers serve a broad range of children at different developmental stages, and the facilities vary widely in size, type, and the number of clients they serve. As such, much of the state guidance recognizes that the adoption of some safety measures may not be feasible for all providers. Even so, many childcare providers have remained open throughout the pandemic to provide an essential service.

\section{Commonalities of Emergency Childcare Guidance Pertinent to Center-Based ABA}

An analysis of state childcare guidance identified variables with potential relevance to the center-based operations of ABA organizations, including recommendations or requirements for daily health screenings of employees and clients, procedures for dropping off and picking up clients, hygiene protocols, use of face coverings, physical distancing, use of personal protective equipment (PPE), maintenance of stable cohorts of clients and employees, safety protocols for meals and snacks, monitoring of absenteeism, specifications for facility cleaning, and signage.

\section{Daily Health Screenings}

Most states require or encourage daily health screenings of both the client and employees. Some states recommend the health screening be applied to all those who reside with the client, and some states recommend screening all those who reside with employees as well. Health screenings are typically comprised of taking temperatures and asking a series of questions, such as those listed in Table 2.

Individuals who respond yes to any of the screening questions are not permitted to enter the center and cannot return until after they have been symptom-free for $72 \mathrm{hr}$ without the aid of fever-reducing medication (i.e., acetaminophen). A great deal of state guidance cautions providers to be attentive 
to emerging symptoms throughout the day and to retake temperatures if children or staff appear flushed or exhibit other signs of illness.

\section{Drop-Off and Pickup Procedures}

The CDC recommends staggering drop-off and pickup times in order to limit the amount of close contact between children, parents/caregivers, and staff members in high-traffic situations and times (CDC, 2020a). Additionally, some states recommend establishing procedures to greet children outside the center and bring them to their parents/caregivers at the end of the day, have children wash their hands immediately upon arrival, and modify sign-in/sign-out procedures to limit unnecessary exchanges and eliminate shared objects, such as pens, pencils, and touch screens. Several states recommend incorporating the health screening before the child enters the center, which would decrease the risk of contaminating the facility and spreading the virus to others if the health screening yields an indication that the child may be ill.

\section{Hygiene}

Hygiene encompasses both the personal hygiene of the clients and employees, including handwashing and covering coughs and sneezes, and the hygienic treatment of toys, technology, and food. Frequent handwashing is among the most important components of preventing the spread of the virus (CDC, 2020b).

Handwashing Clients and staff should be taught proper handwashing techniques, and some states require signage illustrating such techniques to be posted by sinks and in bathrooms (Table 1). Hands should be washed upon arrival at the center and frequently throughout the day. The most current CDC guidance underscores that soap and water are more effective than hand sanitizer, which remains in short supply. Ideally, use of hand sanitizer should be followed by traditional handwashing as soon as possible.

Coughing and sneezing Clients and staff should avoid touching their eyes, nose, and mouth and should use a tissue to cover their mouths and noses when coughing or sneezing. Tissues should be discarded immediately in nearby receptacles that do not hinder social distancing, and hands should be washed after the tissue is discarded.

\section{Toys}

Proper disinfection of toys must occur between clients and at the end of the day. Toys with porous surfaces, such as stuffed animals and dolls with clothing, should be removed from the center. Toys that encourage mouthing, such as play food and utensils, should not be accessible. Items that cannot be disinfected, such as Play-Doh and slime, should not be in the center. Toys that cannot be readily disinfected should be removed. Technology, such as iPads, should not be shared between the client and the clinician and should be disinfected thoroughly before and after its use.

\section{Masks and Face Coverings}

In situations where physical distancing of $6 \mathrm{ft}$ cannot be maintained, the $\mathrm{CDC}$ recommends the use of masks to reduce the risk of exposure. Given the ongoing shortages of N95 respirator masks, they should be reserved for frontline medical personnel, such as doctors and nurses; however, surgical masks or even cloth coverings are recommended. Due to the limited availability of surgical masks and other PPE, organizations are encouraged to reach out to their local health departments or state emergency management agencies to access these supplies.

The use of face shields, although primarily used by medical personnel to protect against spray and splatter, may be effective as they allow the client to see the staff member's full face and facial expressions, but more research is needed to determine their effectiveness in preventing the spread of the virus.

\section{Physical Distancing}

Toward the end of March 2020, the World Health Organization made a point to reframe distancing recommendations between people as physical distancing, rather than social distancing, underscoring that physical and social connections are distinct and that the intent is to encourage physical distancing (World Health Organization, 2020). The CDC recommends that group sizes be limited to 10 people, inclusive of staff and clients, and that $6 \mathrm{ft}$. of distance be maintained between individuals as much as possible. Additionally, the $\mathrm{CDC}$ recommends, wherever possible, stable groups in which the clients and employees remain the same and the number of people working with a client is limited.

Physical-distancing measures can be integrated into a center by limiting smaller rooms to one employee and one client. In centers with large, open spaces, larger pieces of furniture or plexiglass barriers can facilitate physical distancing, and tape on the floor can encourage adherence to physical-distancing recommendations.

\section{Meals and Snacks}

Although meal and snack times are natural opportunities to foster social engagement with peers, organizations should consider having clients eat with their assigned staff in their individual therapy/treatment areas. Parents/caregivers should be discouraged from providing food that requires additional 
preparation and encouraged to send in disposable utensils. Food should be provided in a paper bag. Thorough handwashing should precede and follow all meal and snack times. If common surfaces are touched, they should be disinfected.

\section{Contaminated Clothing}

Clothes contaminated with body fluids or secretions should be placed in a plastic bag or washed in a washing machine (Occupational Safety and Health Administration Bloodborne Pathogens, 1991). Families/caregivers should be encouraged to provide changes of clothes for their children in a quantity sufficient to last a designated time period. Staff members are encouraged to wear layers in the event their clothing becomes soiled, so they can remove the outer/top layer. Alternatively, staff members should have a change of clothes available.

\section{Facility Cleaning}

Specific sanitizing and disinfecting procedures are described in detail in the CDC guidance (CDC, 2020a). Common surfaces that are touched throughout the day, such as doorknobs, light switches, tables, and countertops, should be disinfected repeatedly during the day. Facilities should be sanitized and disinfected thoroughly at the end of each day. ABA organizations should ensure that necessary cleaning supplies are readily available to staff but not accessible to children.

\section{Client Readiness}

\section{Assessing Readiness}

Although the majority of available resources focus on preparing facilities and processes for operation, organizations should also consider the readiness of their clients and clinicians to deliver services consistent with the organization's health and safety plan. The efficacy of the health and safety plan and its protocols is predicated on the ability of staff and clients to follow the necessary precautions. Given the client population served by ABA organizations, clinicians should take great care in understanding and teaching the skills that are necessary for clients to demonstrate safe behaviors as identified in the health and safety plan.

Although there are many commonalities in state guidance, the recommended measures have specific clinical implications for individuals with autism spectrum disorder (ASD). Consistent with their diagnosis, individuals with ASD may present with rigidities around routines or aversions to sensory stimuli, such as masks, that may be exacerbated when transitioning back to center-based services. Preteaching of specific skills via telehealth or in the home setting prior to reentry into the center may increase client and staff compliance with the organization's health and safety plan.
As part of each clinician's decision-making process, clinicians should weigh the considerations listed in Table 3 as they relate to each client's skill set and presentation. These considerations allow a clinician to determine whether the client currently demonstrates the necessary safety skills to be a good fit for center-based services or if preteaching or accommodations would be needed in a given area to ensure that the health and safety plan can be carried out. Colombo, Wallace, and Taylor (2020) address the importance of determining the clinical appropriateness of settings for each client during emergencies (Colombo et al., 2020).

Considering the most relevant state guidance and the organization's health and safety plan, the treatment team should develop a list of required prerequisite skills in order for clients to share space safely. For example, an organization's health and safety plan may identify that clients who will be a part of a group working in the same space should all minimally tolerate wearing masks themselves and tolerate others wearing masks for the session duration. This criterion may be loosened in instances where the space can accommodate one client and staff pairing working in a given area. In those cases, the health and safety plan may identify that clients who do not yet possess the necessary prerequisite skills for group spaces should be prioritized for smaller spaces that are not shared with other clients.

\section{Teaching Readiness}

Practitioners should look to the behavior-analytic research literature for established treatments to teach readiness skills. For example, new pickup and drop-off routines may be taught with graduated guidance and picture schedules (Bryan \& Gast, 2000; Dooley, Wilczenski, \& Torem, 2001) or story-based interventions (Bledsoe, Myles, \& Simpson, 2003; Ivey, Heflin, \& Alberto, 2004). Hygiene skills may be taught via task analysis (Probst \& Walker, 2017), using modeling (Keen, Brannigan, \& Cuskelly, 2007), chaining (Murzynski \& Bourret, 2007), or combinations of other behavioral interventions (Parrott, Schuster, Collins, \& Gassaway, 2000). Desensitization to protective equipment could be achieved through exposure (Ellis, Ala'i-Rosales, Glenn, Rosales-Ruiz, \& Greenspoon, 2006; Koegel, Openden, \& Koegel, 2004). Physical distancing may be shaped using behavioral interventions that include using reinforcement to teach new instructions and task directions (Newman, Hemmes, Buffington, \& Andreopoulos, 1995), video modeling (Ihrig \& Wolchick, 1988), or other stimulus control techniques.

\section{Staff Training and Support}

In addition to completing a comprehensive health and safety plan and determining client readiness, organizations need 
robust staff training on adherence to the new safety procedures, as well as a plan for support mechanisms that will ensure compliance. The safety of clients and staff is paramount, and ABA organizations have the responsibility to use all of their expertise in behavior change to ensure that necessary safety procedures are closely followed. For guidance, there is research in the medical literature on increasing compliance with safety procedures and the use of PPE (Gammon, Morgan-Samuel, \& Gould, 2007; Hinkin, Gammon, \& Cutter, 2008; McGonagle et al., 2016; Seo, Sohng, Chang, Chaung, \& Won, 2019; Stephens \& Ludwig, 2005). Moreover, multitudes of behavior-analytic research studies address changing and maintaining behavior (e.g., Cooper, 2006a, b; Seo et al., 2019; Stephens \& Ludwig, 2005).

Given the fluidity of what is known about COVID-19, organizations must be poised to respond to changing information. As new details about COVID-19 emerge (i.e., risks, prevention methods, safety guidance), organizations must be prepared to change safety procedures and introduce new training and support systems without delay.

\section{Safety Procedures}

Safety policies and procedures should be clear and specific and should address all relevant topics (e.g., daily health screenings, drop-off and pickup procedures, hygiene, PPE, physical distancing, meals, contaminated clothing, and what to do if a child or employee becomes ill while on site). The organization needs to ensure staff receive adequate training on each policy and procedure, using training best practices. Consider using a behavioral skills training format that includes describing the behavior, demonstrating the behavior, and then having the trainee perform the behavior while the trainer gives feedback, repeating until mastery (Fetherston \& Sturmey, 2014; Parsons, Rollyson, \& Reid, 2012)

\section{Management Commitment}

Studies indicate that behavioral safety processes may be followed more consistently when management takes active measures demonstrating its commitment to these processes. Managers' ongoing behavior regarding safety procedures communicates their relative importance to staff. When workers perceive their manager to value safe working behaviors, safe behavior becomes more likely and employee injuries and accidents decrease (McGonagle et al., 2016). Management commitment can be demonstrated by doing things that assist supervisees achieve a goal, such as completing an observation, contacting staff to provide support or to discuss safety performance, holding a feedback meeting, conducting safety trainings, answering questions, or addressing a hazard (Cooper, 2006a, b).

\section{Data Collection}

As with any important behavior change, it is critical that safety procedure compliance be measured so that the efficacy of training and support systems can be continuously evaluated. Organizations may consider adding this data collection to their typical procedural integrity measures. An adequate monitoring system includes training observers to collect reliable data, transferring data to a graphic display, and continually using the system to trigger retraining or remediation when warranted (Sulzer-Azaroff, McCann, \& Harris, 2001).

\section{Supportive Contingencies}

In addition to adequate training, a critical element of encouraging safe behavior is creating contingencies in the working environment that support ongoing compliance with safety protocols. Numerous evidence-based behavioral strategies have been shown to be effective at teaching and maintaining staff compliance with safety procedures. Procedures that have been used to improve staff compliance with safety equipment and protocols include antecedent strategies (e.g., verbal instructions, awareness training, campaigns/reminder posters, provision of supplies, and goal setting) and consequences (e.g., reinforcement, public posting, individual/group feedback, and self-monitoring; Cooper, 2006a, b; Seo et al., 2019; Stephens \& Ludwig, 2005, cited in Gammon et al., 2007).

Reducing potential barriers to the use of PPE and other safety procedures should be considered proactively when designing safety policies and procedures. Noncompliance in the use of PPE and other safety procedures by medical staff has been the subject of a number of research studies; health care workers cite various reasons for noncompliance with PPE use, including its interference with work, inconvenience, being too busy, an insufficient supply of materials, exercising personal judgment regarding patient risk, confusion about the policy, or simple forgetfulness (Gammon et al., 2007; Stephens \& Ludwig, 2005).

Organizations should anticipate and address potential reasons for noncompliance. For instance, masks could interfere with direct therapy by preventing the client from seeing the clinician's facial expression, resulting in low compliance with mask policies. In such a case, an organization may consider using face shields or masks with a transparent window. If cleaning supplies are located in an area that is not convenient, compliance with sanitizing procedures may be lower; to increase compliance, establish safe places for cleaning supplies situated throughout the center. 


\section{Limitations}

As scientists and medical professionals learn more about COVID-19, guidance may change. ABA providers should be sensitive to the fluid nature of this information and should be alert to updates at the state and federal levels.

The authors recognize that staffing challenges may present a barrier to reopening but viewed this topic as outside the scope of this article.

\section{Looking Ahead}

Recognizing that the impact of COVID-19 will not be short lived, ABA providers and the families they serve are encouraged to think in terms of how best to ensure access to ABA in an unpredictable, long-term pandemic. Although facts about COVID-19 continue to emerge, the safety measures it requires will not change until a vaccine is broadly available. For that reason, ABA providers should thoroughly understand how best to minimize the spread of COVID-19 among employees and clients and should endeavor to use all of the tools available to them to maintain or renew access to ABA safely.

To preserve access to ABA, states issued directives that required insurers and health plans to authorize telehealth for all ABA billing codes. For the first time, behavior technicians were permitted to deliver one-to-one ABA via telehealth. In order to preserve these gains, behavior analysts should publish on telehealth's effectiveness, recognizing that, in emergencies, progress may be limited to the prevention of the worsening of delays and maladaptive behaviors.

Recognizing that childcare centers have remained open without interruption, ABA providers and their clients should individualize decisions about treatment locations and the use of telehealth and should resist policies, advocacy, or posturing that creates barriers to accessing ABA over extended periods of time. Even in a pandemic, ABA providers and those they serve should be alert to violations of mental health parity laws and the equitable access to $\mathrm{ABA}$ that they are meant to protect.

\section{Compliance with Ethical Standards}

Conflict of Interest The authors declare that they have no conflict of interest.

Ethical Approval This article does not contain any studies with human participants or animals performed by any of the authors.

\section{References}

Bledsoe, R., Myles, B. S., \& Simpson, R. L. (2003). Use of a social story intervention to improve mealtime skills of an adolescent with Asperger syndrome. Autism: The International Journal of
Research and Practice, 7(3), 289-295. https://doi.org/10.1177/ 1362361303007003005.

Bryan, L. C., \& Gast, D. L. (2000). Teaching on-task and on-schedule behaviors to high-functioning children with autism via picture activity schedules. Journal of Autism and Developmental Disorders, 30(6), 553-567. https://doi.org/10.1023/A:1005687310346.

Centers for Disease Control. (2020a). Guidance for child care programs that remain open. Retrieved from https://www.cdc.gov/coronavirus/ 2019-ncov/community/schools-childcare/guidance-for-childcare. html.

Centers for Disease Control. (2020b). Hand hygiene guidance. Retrieved from https://www.cdc.gov/handhygiene/providers/index.html

Colombo, R. A., Wallace, M., \& Taylor, R. (2020). An essential service decision model for applied behavior analytic providers during crisis. Behavior Analysis in Practice. Advance online publication. Retrieved from https://doi.org/10.31234/osf.io/te8ha.

Cooper, D. (2006a, March). The impact of management's commitment on employee behavior: a field study. Paper presented at the American Society of Safety Engineers Middle East Chapter Seventh Professional Development Conference \& Exhibition, Seef Area, Kingdom of Bahrain.

Cooper, M. D. (2006b). Exploratory analyses of the effects of managerial support and feedback consequences on behavioral safety maintenance. Journal of Organizational Behavior Management, 26(3), 1-31. https://doi.org/10.1300/J075v26n03 01.

Cox, D. J., Plavnick, J. B., \& Brodhead, M. T. (2020). A proposed process for risk mitigation during the COVID-19 pandemic. Behavior Analysis in Practice. Advance online publication. https://doi.org/10. 1007/s40617-020-00430-1.

Dooley, P., Wilczenski, F. L., \& Torem, C. (2001). Using an activity schedule to smooth school transitions. Journal of Positive Behavior Interventions, 3(1), 57-61. https://doi.org/10.1177/ 2F109830070100300108.

Ellis, E. M., Ala'i-Rosales, S. S., Glenn, S. S., Rosales-Ruiz, J., \& Greenspoon, J. (2006). The effects of graduated exposure, modeling, and contingent social attention on tolerance to skin care products with two children with autism. Research in Developmental Disabilities, 27(6), 585-598. https://doi.org/10.1016/j.ridd.2005. 05.009 .

Fetherston, A. M., \& Sturmey, P. (2014). The effects of behavioral skills training on instructor and learner behavior across responses and skill sets. Research in Developmental Disabilities, 35(2), 541-562. https://doi.org/10.1016/j.ridd.2013.11.006.

Gammon, J., Morgan-Samuel, H., \& Gould, D. (2007). A review of the evidence for suboptimal compliance of healthcare practitioners to standard/universal infection control precautions. Journal of Clinical Nursing, 17(2), 157-167. https://doi.org/10.1111/j.1365-2702. 2006.01852.x.

Hinkin, J., Gammon, J., \& Cutter, J. (2008). Review of personal protection equipment used in practice. British Journal of Community Nursing, 13(1), 14-19. https://doi.org/10.12968/bjen.2008.13.1. 27978.

Ihrig, K., \& Wolchick, S. A. (1988). Peer versus adult models and autistic children's learning: Acquisition, generalization, and maintenance. Journal of Autism and Developmental Disorders, 18, 67-79. https://doi.org/10.1007/BF02211819.

Ivey, M. L., Heflin, L., \& Alberto, P. (2004). The use of social stories to promote independent behaviors in novel events for children with PDD-NOS. Focus on Autism and Other Developmental Disabilities, 19(3), 164-176. https://doi.org/10.1177/ 2F10883576040190030401.

Keen, D., Brannigan, K. L., \& Cuskelly, M. (2007). Toilet training for children with autism: The effects of video modeling. Journal of Developmental and Physical Disabilities, 19, 291-303. https:// psycnet.apa.org/doi/10.1007/s10882-007-9044-x. 
Koegel, R. L., Openden, D., \& Koegel, L. K. (2004). A systematic desensitization paradigm to treat hypersensitivity to auditory stimuli in children with autism in family contexts. Research and Practice for Persons With Severe Disabilities, 29(2), 122-134. https://doi.org/ 10.2511/2Frpsd.29.2.122.

McGonagle, A. K., Essenmacher, L., Hamblin, L., Luborsky, M., Upfal, M., \& Arnetz, J. (2016). Management commitment to safety, teamwork, and hospital worker injuries. Journal of Hospital Administration, 5(6), 46-52. https://doi.org/10.5430/jha.v5n6p46.

Murzynski, N. T., \& Bourret, J. C. (2007). Combining video modeling and least-to-most prompting for establishing a response chain. Behavioral Interventions, 22, 147-152. https://doi.org/10.1002/ bin.224.

Newman, B., Hemmes, N. S., Buffington, D. M., \& Andreopoulos, S. (1995). The effects of schedules of reinforcement on instructionfollowing in human subjects with verbal and nonverbal stimuli. Analysis of Verbal Behavior, 12, 31-41. https://doi.org/10.1007/ 2FBF03392895.

Occupational Safety and Health Administration Bloodborne Pathogens, 29 C.F.R. § 1910.1030 (1991). Retrieved from https://www.osha. gov/laws-regs/regulations/standardnumber/1910/1910.1030

Parrott, K. A., Schuster, J. W., Collins, B. C., \& Gassaway, L. J. (2000). Simultaneous prompting and instructive feedback when teaching chained tasks. Journal of Behavioral Education, 10, 3-19. https:// doi.org/10.1023/A:1016639721684.

Parsons, M. B., Rollyson, J. H., \& Reid, D. H. (2012). Evidence-based staff training: A guide for practitioners. Behavior Analysis in Practice, 5(2), 2-11. https://doi.org/10.1007/BF03391819.

Probst, K. M., \& Walker, V. L. (2017). Using the system of least prompts to teach personal hygiene skills to a high school student with comorbid visual impairment and autism spectrum disorder. Journal of Visual Impairment and Blindness, 111(6), 511-525. https://doi. org/10.1177/2F0145482X1711100603.
Seo, H. J., Sohng, K. Y., Chang, S. O., Chaung, S. K., \& Won, J. S. (2019). Interventions to improve hand hygiene compliance in emergency departments: A systematic review. Journal of Hospital Infection, 102(4), 394-406. https://doi.org/10.1016/j.jhin.2019.03. 013.

Stephens, S. D., \& Ludwig, T. D. (2005). Improving anesthesia nurse compliance with universal precautions using group goals and public feedback. Journal of Organizational Behavior Management, 25(2), 37-71. https://doi.org/10.1300/J075v25n02_02.

Sulzer-Azaroff, B., McCann, K. B., \& Harris, T. C. (2001). The safe performance approach to preventing job-related illness and injury. In C. M. Johnson, W. K. Redmon, \& T. C. Mawhinney (Eds.), Handbook of organizational performance: Behavior analysis and management (pp. 289-290).

Sun, L. H. (2020, April 21). CDC director warns second wave of coronavirus is likely to be even more devastating. Washington Post. Retrieved from https://www.washingtonpost.com/health/2020/04/ 21/coronavirus-secondwave-cdedirector/.

Taylor, M. A., \& Alvero, A. M. (2012). The effects of safety discrimination training and frequent safety observations on safety-related behavior. Journal of Organizational Behavior Management, 32(3), 169-193. https://doi.org/10.1080/01608061.2012.698115.

U.S. Constitution, amend. X.

World Health Organization. (2020, March 20). World health organization transcript of press conference. Retrieved from https://www.who.int/ docs/default-source/coronaviruse/transcripts/who-audioemergencies-coronavirus-press-conference-full-20mar2020.pdf? sfvrsn=1 eafbff 0

Publisher's Note Springer Nature remains neutral with regard to jurisdictional claims in published maps and institutional affiliations. 